\title{
Design of miniaturized dual-mode microstrip bandpass filter based on a novel fractal resonator
}

\author{
Hong-Shu Lu ${ }^{\dagger}$, Tao Xie, Jing-Jian Huang and Nai-Chang Yuan \\ College of Electronic Science and Engineering, \\ National University of Defense Technology, Changsha, 410073, China \\ †E-mail: luhongshu0321@163.com
}

\begin{abstract}
In this letter, a fractal structure used for the miniaturized design of microstrip bandpass filter (BPF) is presented. A new resonator called Greek cross fractal resonator (GCFR) in this paper was constructed by combining the proposed fractal structure with a traditional dual-mode meandered loop resonator. Three BPFs based on the new resonator with different orders iteration were analyzed. In addition, the mode-splitting characteristic and impact of the perturbation element on the performance of the BPFs were also investigated. The simulated results show that the new fractal resonator has a certain potential in the miniaturization design and the BPFs possess acceptable frequency characteristics. Therefore, the proposed fractal structure can be used as a candidate for miniaturization technology.

Keywords: Dual-mode; Microstrip bandpass filter (BPF); Miniaturization; Greek cross
\end{abstract} fractal resonator.

\section{Introduction}

Microstrip bandpass filters (BPFs), as one of the indispensable parts in the RF front ends of various wireless communication systems, play an important role in filtrating the signals in the specific frequency band and restraining the clutter and disturb signals. Consequently, designing microstrip BPFs is always a highly active research area in wireless communication systems. With the rapid development of modern wireless communication technology, the market demand for microstrip BPFs with excellent performance and compact structure is becoming stronger and stronger.

Since the dual-mode resonator is firstly introduced by Wolff [1], a lot of research on the dual-mode resonators has been carried out [2-4]. In these resonators, the resonators are composed of two degenerate modes and excited by asymmetrical feed lines or adding a perturbation element in one of the resonator corners. The main advantage of the dual-mode resonator is that it can be used as a doubly tuned resonator circuit, therefore, the number of resonators required for 
a given degree filter is reduced by half, resulting in a compact filter configuration.

Fractal was first defined by Mandelbrot as a method of assorting structures whose dimensions were not complete numbers and had broken or irregular fragments [5]. Different from the Euclidean geometries, fractal geometries have two common properties: space-filling and self-similarity, which are attractive for microwave, semiconductor, and MEMS designers. It has been shown that using the space-filling property can effectively reduce the physical size of the antenna and the self-similarity property has been successfully applied to design multiband antennas [6]. One of the pioneer research work in the prediction of the use of fractal geometry in filter design is that of Yordanov et al [7]. Since then, research work in this field has shown a dramatic increase. A variety of fractal geometries have been applied to design compact BPFs [8-10].

In this paper, a new fractal resonator applied to design miniaturized microstrip BPF is presented. By increasing the iteration order of the proposed resonator, the resonant frequency shifts towards the lower frequency. Therefore, the proposed fractal structure possesses the miniaturized property. A demonstrated BPF with second iteration GCFR is modeled and analyzed to discuss the mode-splitting characteristic and the impact of the perturbation element on the performance of the BPF.

\section{Generation Process of Greek Cross Fractal Resonator}

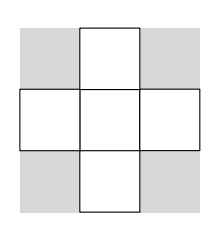

(a)

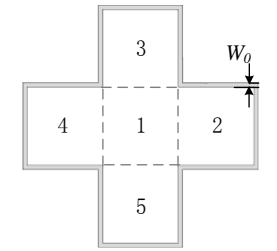

(b)

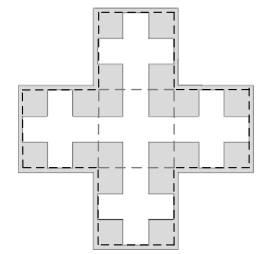

(c)

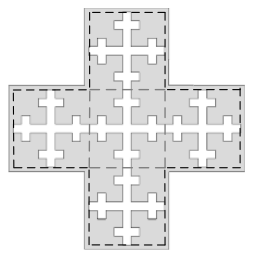

(d)

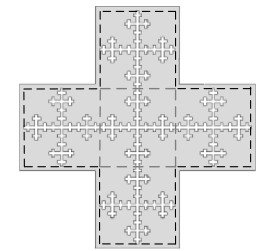

(e)

Fig. 1. Generation process of GCFR. (a) the generator. (b) the initiator: meandered loop resonator. (c) first iteration. (d) second iteration. (e) third iteration.

The starting pattern for the generation process is a traditional meandered loop resonator, as shown in Fig. 1(b). In order to start this type of fractal structure, the 
internal cavity of the initiator is divided into five small congruent squares besides the narrow strip with a uniform width W0 located on the edge of the periphery. Then, each of these five small squares is replaced and etched by what is called the generator shown in Fig. 1(a). The generator will be scaled to $1 / 3$ when the last iteration is completed so that the etched portions can be seamlessly stitched together. In order to explain the generation process, the first three iterations of GCFR are depicted in Fig. 1(c), (d) and (e) respectively.

\section{Design of Dual-mode BPF}

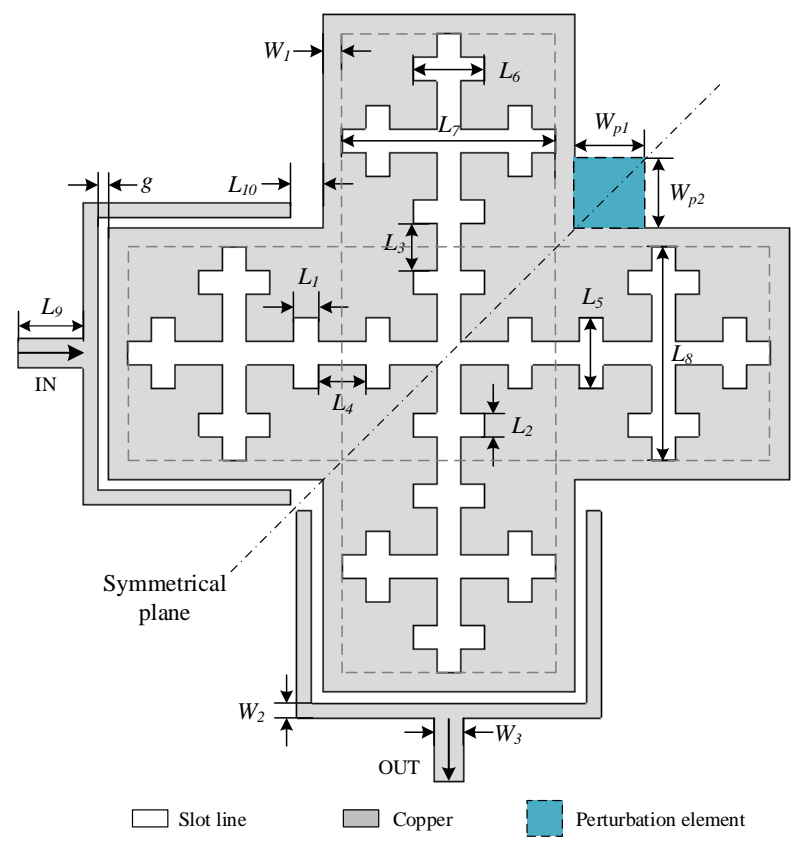

Fig. 2. Configuration of the proposed BPF.

Two $50 \Omega$ feed lines as input and output (I/O) ports are spatially separated orthogonally. The resonator is excited by using an edge spacing coupling method to I/O ports. A perturbation element is located at one corner of the GCFR which is $135^{\circ}$ offset from both input and output ports, as depicted in Fig. 2. Thus, the dual-mode BPF is established.

For comparison, a dual-mode BPF based on the meandered loop resonator, and three dual-mode BPFs based on the GCFR with different orders iteration are simulated by using the CST Microwave Studio 2014 electromagnetic simulator. It has been supposed that, these filter configurations have been etched on a substrate with a relative dielectric constant of 10.8, dielectric thickness of 1.27 $\mathrm{mm}$ and metallization thickness of $35 \mu \mathrm{m}$. The conductor material is assumed to 
be perfect conductor. All the resonators are approximately equal, and the detail dimensions of the four resonators are listed in Table 1.

Table 1. Dimensions of BPF with different orders iteration

\begin{tabular}{ccccc}
\hline Dimensions [mm] & Initiator & First iterator & Second iterator & Third iterator \\
\hline$L_{1}=L_{2}$ & & & 0.6 & 0.6 \\
$L_{3}=L_{4}$ & & & 1.2 & 1.2 \\
$L_{5}=L_{6}$ & & 1.8 & 1.8 & 1.8 \\
$L_{7}=L_{8}$ & 5.4 & 5.4 & 5.4 & 5.4 \\
$L_{9}$ & 2 & 2 & 2 & 2 \\
$L_{10}$ & 1 & 0.95 & 0.9 & 0.75 \\
$W_{1}$ & 0.6 & 0.43 & 0.3 & 0.52 \\
$W_{2}$ & 0.2 & 0.2 & 0.4 & 0.32 \\
$W_{3}$ & 0.98 & 0.98 & 0.98 & 0.98 \\
$W_{\mathrm{p} 1}=W_{\mathrm{p} 2}$ & 2.4 & 2.35 & 2.15 & 2.3 \\
$\mathrm{~g}$ & 0.3 & 0.2 & 0.2 & 0.26 \\
\hline
\end{tabular}

\section{Performance Evaluation}

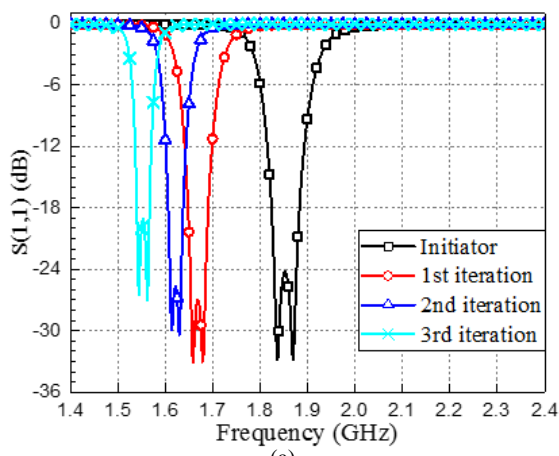

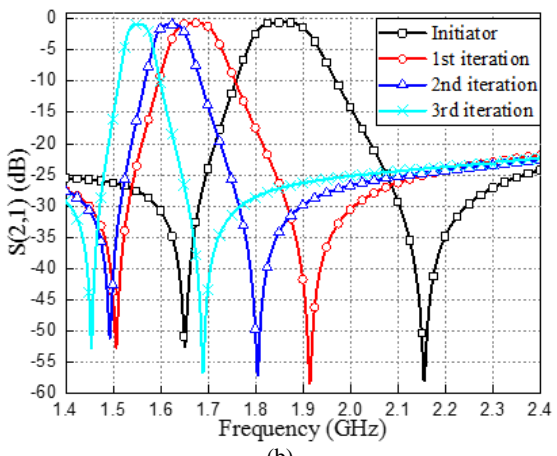

(b)

Fig. 3. Frequency responses of BPFs with different orders iteration.(a) the return loss responses. (b) the transmission responses.

Table 2. Frequency responses of BPFs with different orders iteration

\begin{tabular}{|c|c|c|c|c|c|}
\hline \multicolumn{2}{|l|}{ Simulation } & Initiator & First iterator & Second iterator & Third iterator \\
\hline \multicolumn{2}{|l|}{$f_{0}[\mathrm{GHz}]$} & 1.85 & 1.67 & 1.62 & 1.56 \\
\hline \multicolumn{2}{|l|}{$-3 \mathrm{~dB}$ BW [MHz] } & 122 & 92 & 69 & 51 \\
\hline \multicolumn{2}{|l|}{ FBW [\%] } & 6.49 & 5.51 & 4.26 & 3.27 \\
\hline \multicolumn{2}{|c|}{ Min insertion loss $[\mathrm{dB}]$} & 0.59 & 0.68 & 0.89 & 0.9 \\
\hline Resonance & Mode I & 1.84 & 1.66 & 1.61 & 1.55 \\
\hline frequency $[\mathrm{GHz}]$ & Mode II & 1.87 & 1.68 & 1.63 & 1.57 \\
\hline Transmission & Lower & -52.6 & -52.6 & -51.1 & -52.9 \\
\hline zeros $[\mathrm{dB}]$ & Upper & -58 & -58.4 & -57.2 & -57.2 \\
\hline \multicolumn{3}{|l|}{ Size reduction [\%] } & 10.8 & 13.8 & 17.4 \\
\hline
\end{tabular}


The return loss and transmission responses of the four BPFs are shown in Fig. 3(a) and (b), respectively. It is clear that all BPFs have a quasi-elliptic frequency response, each of the four filters has two transmission zeros located on both sides of the passband. The corresponding simulated results of frequency response are tabulated in Table 2. From the simulated results, it can be seen that the proposed fractal structure possesses the miniaturized property. Therefore, the new fractal resonator can be applied to design compact microwave components.

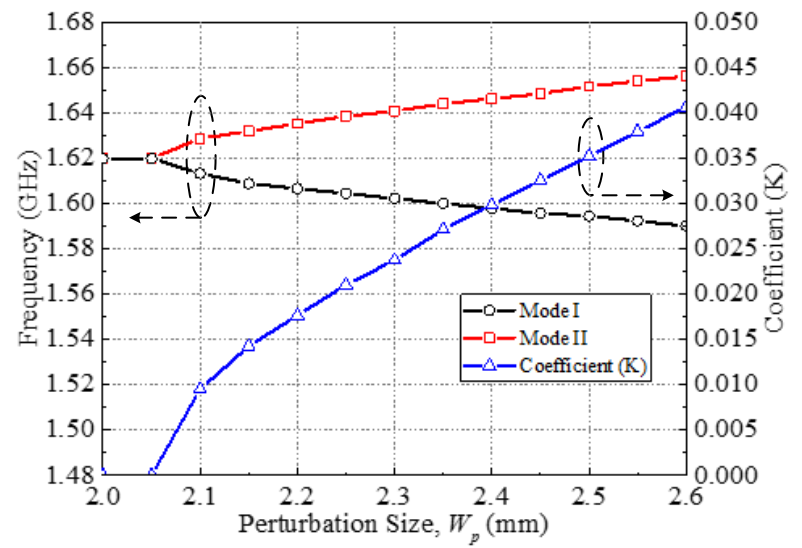

Fig. 4. Resonance frequencies of degenerate modes and coupling coefficient against the perturbation size $W_{\mathrm{p}}$.

Fig. 4 plots the performance of mode splitting with the perturbation size changing from $2 \mathrm{~mm}$ to $2.6 \mathrm{~mm}$. As it can be seen that, when $W_{\mathrm{p}} \geq 2.1 \mathrm{~mm}\left(W_{\mathrm{p} 1}\right.$ $=W_{\mathrm{p} 2}=W_{\mathrm{p}}$ ), both degenerate modes appear and with the increasing of the perturbation size, the degree of the resonance frequency splitting is more serious and accordingly the bandwidth of the passband expands.

The coupling coefficient, denoted by $K$, between the degenerate modes can be calculated as follows [11]:

$$
K=\frac{f_{02}^{2}-f_{01}^{2}}{f_{02}^{2}+f_{01}^{2}}
$$

where $f_{01}$ and $f_{02}$ indicate the respective resonant frequency of Mode I and Mode II. It is apparent that the coupling coefficient increases with the increasing of the perturbation size, as shown in Fig. 4.

\section{Conclusions}

A new fractal resonator applied to design miniaturized microstrip BPF is introduced in this paper. Compared with the traditional meandered loop resonator, by increasing the iteration order of GCFR from 1 to 3 , the resonance 
frequency shifts from $1.85 \mathrm{GHz}$ to $1.56 \mathrm{GHz}$, which indicates the proposed fractal resonator possesses the miniaturized property. Additionally, in order to discuss the mode-splitting characteristic and the impact of the perturbation element on the performance of the BPF, a demonstrated BPF based on the GCFR with second iteration is analyzed. The simulated results show that with the increasing of the perturbation size, two degenerate modes are excited and the degree of the resonance frequency splitting is more serious. What's more, the coupling coefficient grows in pace with the perturbation size.

\section{References}

1. I. Wolff, Microstrip bandpass filter using degenerate modes of a microstrip ring resonator, Electronic letters 8, 302 (1972).

2. C. Karpuz, A.K. Gorur, and E. Sahin, Dual-mode dual-band microstrip bandpass filter with controllable center frequency, Microwave and Optical Technology Letters 57, 639 (2015).

3. E.S. Ahmed, Dual-mode dual-band microstrip bandpass filter based on fourth iteration T-square fractal and shorting pin, Radioengineering 21, 617 (2012).

4. J.-W. Baik, L. Zhu, and Y.-S. Kim, Dual-mode dualband bandpass filter using balun structure for single substrate configuration, IEEE Microwave and Wireless Components Letters 20, 613 (2010).

5. B.B. Mandelbrot, The Fractal Geometry of Nature (W. H. Freeman and Company, 1983)

6. J.K. Ali, A new reduced size multiband patch antenna structure based on Minkowski perfractal geometry, Journal of Engineering and Applied Sciences 2, 1120 (2007).

7. O.I. Yordanov, et al. Prospects of fractal filters and reflectors, in 1991 Seventh International Conference on (IEE) Antennas and Propagation (ICAP 91) (New York, USA, 1991).

8. C.S. Ye, et al., Resonant properties of the Sierpinski-based fractal resonator and its application on low-loss miniaturized dual-mode bandpass filter, Microwave and Optical Technology Letters 51, 1358 (2009).

9. J.-C. Liu, et al., A novel Minkowski-island-based fractal patch for dualmode and miniaturization band-pass filters, Microwave and Optical Technology Letters 53, 594 (2011).

10. N. Jankovic, R. Geschke, and V. Crnojevic-Bengin, Compact tri-band bandpass and bandstop filters based on Hilbert-Fork resonators, IEEE Microwave and Wireless Components Letters 23, 282 (2013).

11. J.-S. Hong and M.J. Lancaster, Microstrip filters for RF/Microwave applications (John Wiley \& Sons, 2001) 